\title{
Anti-Müllerian Hormone (AMH) is A Good Predictor for Ongoing Pregnancy in Women Undergoing IVF/ICSI in Antagonist Cycles
}

\author{
Negjyp Sopa* \\ The Fertility Clinic, Copenhagen University Hospital, Denmark
}

Submission: January 02, 2019 ; Published: January 10, 2019

*Corresponding author: Negjyp Sopa, The Fertility Clinic, Hvidovre Hospital, Copenhagen University Hospital, Denmark

\begin{abstract}
Observation cohort study, assessed pregnancy rate based only on the AMH algorithm in 888 patients/ 2148 cycles in antagonist protocol for In vitro-fertilisering/intracytoplasmatisk spermainjektion (IVF/ICSI). Patients are divided into 3 groups based on the value of AMH. The first group had AMH <12pmol/L group two between 12-32pmol/L and group three $>32 \mathrm{pmol} / \mathrm{L}$ Patients with low AMH <12pmol/L (n=291 patients/764 cycles) had maximal stimulation with corifollitropin-alfa ( $\mathrm{n}=473 / 62 \%)$ or $300 \mathrm{IU} /$ day of $\mathrm{rFSH}(\mathrm{n}=289 / 38 \%)$ as well $300 \mathrm{IU} /$ day of HP-HMG(n=2/0,3\%). Patients with AMH $12-32 \mathrm{pmol} / \mathrm{L}(\mathrm{n}=401$ patients/941 cycles) had standard stimulation with $150 \mathrm{IU} /$ day of rFSH ( $\mathrm{n}=789 / 84 \%)$, or HP-HMG $150 \mathrm{IU} /$ day $(\mathrm{n}=103 / 11 \%)$ as well corifollitropin-alfa $(\mathrm{n}=49 / 5 \%)$. Patients with AMH $>32 \mathrm{pmol} / \mathrm{L}(\mathrm{n}=196$ patients/ 443 cycles) had minimal stimulation with 112 IU/day of HP-hMG $(\mathrm{n}=244 / 55 \%)$ or rFSH 112 IU/day ( $\mathrm{n}=199 / 45 \%)$. The ongoing pregnancy rates per started cycle in the three AMH groups first cycle were $15 \%, 25 \%$ and $38 \%$ respectively. The chance of achieving an ongoing pregnancy was reduced in women with low AMH whereas high AMH was associated with an increased chance of achieving an ongoing pregnancy, regardless of the patient's age. The proportion with pregnancy in the low AMH group was significantly ( $p<0.001)$ lower than in the high AMH group. The conclusion is that among high AMH patients, has statistically significantly high ongoing pregnancy rate. However, a randomised study with enough power with our protocol should be performed.
\end{abstract}

Keywords: Anti-Müllerian hormone; Antral Follicle Count; IVF/ICSI; Pregnancy rate; Ovarian reserve

Abbreviatations: AMH: Anti-Müllerian hormone; AFC: Antral Follicle Count

\section{Introduction}

Anti-Mullerian hormone (AMH) is a member of the TGF-beta family and is expressed by the small preantral and early antral follicles. The AMH level reflects the size of the primordial follicle pool and the best biochemical marker of ovarian function across an array of clinical situations [1]. In adult women, AMH levels gradually decline as the primordial follicle pool declines with age [2]. The AMH level appears to be an early, reliable, direct indicator of declining ovarian function. In patients planning IVF, an AMH level correlate with the number of oocytes retrieved after stimulation, and is the best biomarker for predicting poor and excessive ovarian response $[3,4]$.

Unlike the day 3FSH, AMH can be measured anytime during the menstrual cycle and typically demonstrates minimal intracycle variability since the growth of small preantral follicles that express it is continuous, not cyclical. Reliable AMH kits [5] are readily available from multiple vendors and $\mathrm{AMH}$ measurements can be obtained from large clinical reference laboratories $[6,7]$.
Anti-Müllerian hormone (AMH) is a very important parameter for adequate ovarian stimulation for women undergoing IVF/ ICSI treatment [8-10]. A multicentre observational Japanese study has shown that AMH had a significantly higher predictive value for normal and high respondents than FSH and E2 [11]. A prospective cohort study suggested that Anti-Müllerian hormone (AMH) is strongly associated with live birth rates after IVF/ICSI [12]. The number of oocytes in a linear increase depends on an AMH level [13].

We implemented the use of Anti-Müllerian hormone (AMH) as a biomarker for ovarian stimulation [14-16] since 2014. Our target was to achieve around 10 oocytes, with a range of 8-14. This was done by segmenting the patients into 3 groups given the maximum, standard or low dose of FSH stimulation according to their AMH levels.

\section{Materials and Methods}

Observation cohort study, where data were retrospectively collected, by 888 patients undergoing their 2148 cycle antagonist 
protocol, at the Fertility Clinic Rigshospitalet, Copenhagen University Hospital from January 2014 to June 2017. All included patients were stimulated according to an AMH-based FSH dosing-algorithm.

Patients with low AMH <12pmol/L (n=291 patients/764 cycles were stimulated with a weight-adjusted dose of Elonva ${ }^{\circledR}$ ( $\mathrm{n}=473 / 62 \%$ ) according to labelling: women $>60 \mathrm{~kg}$ received $150 \mu \mathrm{g}$ and women $<60 \mathrm{~kg}$ received $100 \mu \mathrm{g}$, or $300 \mathrm{IU} /$ day of $\mathrm{rFSH}$ ( $n=289 / 38 \%)$ as well $300 \mathrm{IU} /$ of HP-HMG $(\mathrm{n}=2 / 0,3 \%)$. Patients with AMH 12-32pmol/L ( $\mathrm{n}=401$ patients/941 cycles) had standard stimulation with 150IU/day of $\mathrm{rFSH}$ (follitropin-alfa or -beta) ( $n=789 / 84 \%)$, or HP-HMG 150 IU/day ( $n=103 / 11 \%$ ) as well corifollitropin-alfa $(n=49 / 5 \%)$. Patients with AMH >32pmol/L (n=196 patients/ 443 cycles) were stimulated with Menopur® (HP-hMG) 112 IU/day (n=244/55\%) or rFSH (follitropin-alfa or -beta)112 IU/day ( $n=199 / 45 \%)$. The FSH dose was fixed the first seven days of stimulation. After seven days of stimulation, the FSH dose could be adjusted based on sonographic assessment. The standard criteria for triggering of ovulation were at least 3 follicles $\geq 17 \mathrm{~mm}$.

Oocyte retrieval was done 36 hours after Ovitrelle (hCG)/ Suprefact $₫ \mathrm{GnRH}$ triggering, and a transfer was done at day 2 or day 5. Luteal support was given using vaginal progesterone Lutinus $\AA 100 \mathrm{mg} 3$ times daily for 2 weeks. Lutinus $\AA^{\circledR}$ All data were prospectively collected and recorded in an SSPS version 22 and STATA 13.1. database. The study was approved by the Danish Patient Safety Authority (Number 3-3013-2242/1).

\section{Result}

Baseline characteristics of the 888 patients treated in antagonist protocol in 2148 cycle were stimulated according to an AMH-based FSH dosing algorithm Table 1. As seen, 291 (32.8\%) were in the low AMH group, 401 (45.1\%) in the intermediate and $196(22.1 \%)$ in the high AMH group. Age, AMH and AFC were significantly different between AMH-groups $(\mathrm{p}<0.001)$, whereas weight and BMI are not significant.

Table 1: Baseline characteristics of $888 \mathrm{AMH}$ based hormone stimulation in their first IVF/ICSI cycle.

\begin{tabular}{|c|c|c|c|c|c|}
\hline Serum AMH (Pmol/L) Groups & $<12$ & $12-32$ & $>32$ & All & P- Value \\
\hline Patients, n(\%) & 291(32.8) & $401(45.1)$ & $196(22.1)$ & $888(100)$ & \\
\hline Cycles, n(\%) & $764(35.6)$ & $941(43.8)$ & $443(20.6)$ & $2148(100)$ & \\
\hline Age (Years) mean \pm SD & $35.0 \pm 3.7$ & $32.4 \pm 4.3$ & $31.7 \pm 4.1$ & $33.1 \pm 4.2$ & 0.001 \\
\hline AMH (Anti-Müllerian horm.) mean \pm SD & $6.5 \pm 2.9$ & $20.2 \pm 5.5$ & $57.7 \pm 27,2$ & $24.1 \pm 23.2$ & 0 \\
\hline AFC (Antral follicle count) mean \pm SD & $8.4 \pm 4.7$ & $19.4 \pm 6.8$ & $40.3 \pm 13.6$ & $20.4 \pm 14.2$ & 0 \\
\hline Weight $(\mathrm{kg})$ mean \pm SD & $66.2 \pm 12.1$ & $63.0 \pm 11.6$ & $63.8 \pm 10.4$ & $65.4 \pm 11.6$ & 0.457 \\
\hline BMI $\left(\mathrm{kg} / \mathrm{m}^{2}\right)$ mean \pm SD & $23.5 \pm 3.8$ & $23.4 \pm 3.7$ & $22.6 \pm 3.2$ & $23.1 \pm 3.7$ & 0.474 \\
\hline \multicolumn{6}{|c|}{ Causes of infertility } \\
\hline Anovulation, n(\%) & $9(3.1)$ & $22(5.5)$ & $65(33.2)$ & $96(10.8)$ & \\
\hline Tubal factor, n(\%) & $16(5.5)$ & $27(6.7)$ & $6(3.0)$ & $49(5.5)$ & \\
\hline Uterine factor, $\mathrm{n}(\%)$ & $8(2.7)$ & $8(2.0)$ & $1(0.5)$ & $17(1.9)$ & \\
\hline Endometriosis, n(\%) & $20(6.9)$ & $21(5.2)$ & $6(3.0)$ & $47(5.3)$ & \\
\hline Single women, n(\%) & $45(15.5)$ & $47(11.7)$ & $16(8.2)$ & $108(12.2)$ & \\
\hline Male factor, $\mathrm{n}(\%)$ & $105(36.1)$ & $181(45.2)$ & $75(38.3)$ & $361(40.6)$ & \\
\hline Unexplained, n(\%) & $88(30.2)$ & $95(23.7)$ & $27(13.8)$ & $210(23.7)$ & \\
\hline
\end{tabular}

Data are described with means and standard deviation, Kruskal-Wallis test and median test.

P-value $<0.05$ was considered statistically significant. Statistics, comment in text.

Table 2: Cycle characteristics and outcome in relation to the AMH-based algorithm first cycle IVF/ICSI, in antagonist protocol.

\begin{tabular}{|c|c|c|c|c|c|}
\hline Serum-AMH, Groups & $<12$ & $12-32$ & $>32$ & All & P-Value \\
\hline Patients, n(\%) & $291(32.8)$ & $401(45.1)$ & $196(22.1)$ & $888(10)$ & \\
\hline Elonva(Corifollitropin-alfa) & $203(69.8)$ & $13(3.2)$ & $0(0)$ & $216(24.3)$ & \\
\hline Gonal F or Puregon(rFSH) & $86(29.5)$ & $327(81.6)$ & $26(13.3)$ & $439(49.5)$ & \\
\hline Menopur(hMG) & $2(0.7)$ & $61(15.2)$ & $170(86.7)$ & $233(26.2)$ & \\
\hline Stimulation duration (days) mean $\pm S D$ & $8.5 \pm 1,7$ & $8.5 \pm 1.4$ & $9.5 \pm 2.1$ & $8.7 \pm 1.7$ & 0.004 \\
\hline Cycles converted to IUI, n (\%) & $3(1.0)$ & $5(1.2)$ & $2(1.0)$ & $10(1.1)$ & 0.953 \\
\hline Oocyte retrievals, patients, $\mathrm{n},(\%)$ & $288(99.0)$ & $396(98.8)$ & 194(99.0) & $878(98.9)$ & 0.953 \\
\hline Number of follicles aspirated, mean \pm SD & $5.9 \pm 3.3$ & $9.2 \pm 4.2$ & $9.8 \pm 5.3$ & $8.2 \pm 4.5$ & 0 \\
\hline Number of retrieved oocytes, mean \pm SD & $4.9 \pm 3.2$ & $7.8 \pm 3.8$ & $8.0(4.5)$ & $6.9 \pm 4.1$ & 0 \\
\hline Embryo transfer, $\mathrm{n}(\%$ of oocyte retrievals) & $214(73.5)$ & $338(84.3)$ & $157(80.1)$ & $709(79.8)$ & 0.002 \\
\hline
\end{tabular}




\section{Journal of Gynecology and Women's Health}

\begin{tabular}{|c|c|c|c|c|c|}
\hline Dual transfers, $\mathrm{n}(\%)$ & $32(11)$ & $33(8.2)$ & $10(5.1)$ & $75(8.4)$ & 0.704 \\
\hline Embryos cryopreserved, mean \pm SD (range) & $0.3 \pm 0.4$ & $0.4 \pm 0.5$ & $0.4 \pm 0.5$ & $0.3 \pm 0.5$ & \\
\hline Positive hCG, n (\% of started cycles) & $70(24.1)$ & $148(36.9)$ & $88(44.9)$ & $306(34.5)$ & 0.001 \\
\hline Positive hCG, $\mathrm{n}(\%$ of transfer $)$ & $70(32.7)$ & $148(43.8)$ & $88(56.1)$ & $306(43.2)$ & 0.001 \\
\hline Ongoing pregnancies, $\mathrm{n}(\%$ of started cycles) & $44(15.1)$ & $100(24.9)$ & $75(38.3)$ & $219(24.7)$ & 0.001 \\
\hline Ongoing pregnancies, $\mathrm{n}(\%$ of transfer $)$ & $44(20.6)$ & $100(29.6)$ & $75(47.8)$ & $219(30.9)$ & 0.001 \\
\hline Twins, $n(\%$ ongoing pregnancies $)$ & $4(1.4)$ & $0(0.0)$ & $0(0.0)$ & $4(0.5)$ & \\
\hline Ovarian hyperstimulation syndrome (OHSS), n (\%) & $0(0)$ & $2(0.5)$ & $5(2.5)$ & $7(0.8)$ & 0.028 \\
\hline
\end{tabular}

1. Data are described with means and standard deviation, Kruskal-Wallis test and Chi-square test.

2. Statistics, comment in text.

Table 2 shows first cycle response parameters and clinical outcome in the three AMH groups. The ongoing pregnancy rates per started cycle in the three AMH groups were 15\%,25\% and $38 \%$, respectively $(\mathrm{p}<0.001)$ and per transfer $21 \%, 30 \%$ and

$48 \%$ respectively $(\mathrm{p}<0.001)$. The chance of achieving an ongoing pregnancy was reduced in women with low AMH, whereas high $\mathrm{AMH}$ was associated with an increased chance of achieving an ongoing pregnancy.

Table 3: Cycle characteristics and outcome in relation to the AMH-based algorithm first cycle IVF/ICSI, in antagonist protocol.

\begin{tabular}{|c|c|c|c|c|c|}
\hline Serum-AMH, Groups & $<12$ & $12-32$ & $>32$ & All & P-Value \\
\hline Cycles, n (\%) & $764(35.6)$ & $941(43.8)$ & $443(20.6)$ & $2148(100)$ & \\
\hline Stimulation duration (days) mean \pm SD & $8.5 \pm 1,7$ & $8.6 \pm 1.5$ & $9 . .2 \pm 1.9$ & $8.7 \pm 1.7$ & 0 \\
\hline Elonva & $473(61.9)$ & $49(5.2)$ & $0(0)$ & $522(24.3)$ & \\
\hline Gonal F or Puregon & $289(37.8)$ & $789(83.9$ & $199(44.9)$ & $1277(59.4)$ & \\
\hline Menopur & $2(0.3)$ & 103(10.9) & $244(55.1)$ & $349(16.3)$ & \\
\hline Cycles converted to IUI, n (\%) & $12(1.6)$ & $7(0.7)$ & $7(1.6)$ & $26(1.2)$ & 0.217 \\
\hline Number of follicles aspirated, mean $\pm S D$ & $6.0 \pm 3.4$ & $9.5 \pm 3.9$ & $10.3 \pm 4.9$ & $8.4 \pm 4.4$ & 0 \\
\hline Number of retrieved oocytes, mean \pm SD & $5.1 \pm 3.3$ & $8.1 \pm 3.8$ & $8.6(4.4)$ & $7.2 \pm 4.1$ & 0 \\
\hline Oocyte retrievals, patients, $\mathrm{n},(\%)$ & $746(97.6)$ & 933(99.1) & $436(98.4)$ & $2119(98.6)$ & 0.042 \\
\hline Embryo transfer, $\mathrm{n}$ (\% of oocyte retrievals) & $558(73.0)$ & $796(84.6)$ & $368(84.4)$ & $1722(80.2)$ & 0.001 \\
\hline Dual transfers, $\mathrm{n}(\%)$ & $156(20.4)$ & $179(19.1)$ & $74(16.9)$ & $409(19.0)$ & 0.013 \\
\hline Embryos cryopreserved, mean \pm SD (range) & $0.1 \pm 0.1$ & $0.3 \pm 0.5$ & $0.4 \pm 0.5$ & $0.3 \pm 0.5$ & 0.003 \\
\hline Positive hCG, n (\% of started cycles) & $177(23.2)$ & $327(34.8)$ & $182(41.1)$ & $686(31.9)$ & 0.001 \\
\hline Positive hCG, $\mathrm{n}$ (\% of transfer) & $177(31.7)$ & $327(41.1)$ & $182(49.5)$ & $686(39.8)$ & 0.001 \\
\hline Ongoing pregnancies, n (\% of started cycles) & $110(14.4)$ & $208(22.1)$ & $140(31.6)$ & $458(21.3)$ & 0.001 \\
\hline Ongoing pregnancies, $\mathrm{n}$ (\% of transfer) & $110(19.7)$ & $208(26.1)$ & $140(38.1)$ & $458(26.6)$ & 0.008 \\
\hline Twins, n (\% ongoing pregnancies) & $9(1.2)$ & $5(0.5)$ & $9(2.0)$ & $23(1.1)$ & 0.041 \\
\hline Ovarian hyperstimulation syndrome (OHSS), n (\%) & $0(0)$ & $3(0.3)$ & $11(2.5)$ & $14(0.7)$ & 0.001 \\
\hline
\end{tabular}

1. Data are described with means and standard deviation, Chi-square test, Kruskal-Wallis test one-way ANOVA

2. Statistics, comment in text.

Table 3 shows response parameters and clinical outcome for all cycles 2148. The ongoing pregnancy per started cycle in the AMH groups were $14 \%, 22 \%$ and $32 \%$ respectively $(\mathrm{p}<0.001)$ and per transfer $20 \%, 26 \%$ and 38 respectively $(<0.001)$. In the

pregnancy rate is low after the first cycle, but if we are based on the AMH group, there is still significant pregnancy rate between AMH groups. The pregnancy rate is high in the group with high $\mathrm{AMH}$ and low in the group with low AMH. first cycle, the pregnancy rate is high compared to all cycles. The Table 4: Ongoing pregnancies, number and percentages of started cycles, and of transfer in relation to cycle number and age.

\begin{tabular}{|c|c|c|c|c|c|}
\hline Serum -Amh, Groups & $<12 \mathrm{Pmol} / \mathrm{L}$ & 12-32 Pmol/L & $>32 \mathrm{Pmol} / \mathrm{L}$ & All & P-Value \\
\hline Cycle, $n=1$ & 291(32.8) & $401(45.1)$ & $196(22.1)$ & $888(100)$ & \\
\hline Age (Years) mean $\pm S D$ & $35.0 \pm 3.7$ & $32.4 \pm 4.3$ & $31.7 \pm 4.1$ & $33.1 \pm 4.2$ & 0.001 \\
\hline Ongoing pregnancies, n (\% of started cycles) & $44(15.1)$ & $100(24.9)$ & $75(38.3)$ & $219(24.7)$ & 0.001 \\
\hline Ongoing pregnancies, $\mathrm{n}$ (\% of transfer) & $44(20.6)$ & $100(29.6)$ & $75(47.8)$ & $219(30.9)$ & 0.001 \\
\hline Age (Years) $<35$ mean \pm SD & $30.9 \pm 2.6$ & $29.9 \pm 2.9$ & $29.6 \pm 2.6$ & $30.0 \pm 2.8$ & 0.17 \\
\hline Age $<35$ Ongoing pregnancies, n (\% of started cycles) & $21(19.5)$ & $73(27.4)$ & $53(38.1)$ & $147(28.7)$ & 0.005 \\
\hline
\end{tabular}


Journal of Gynecology and Women's Health

\begin{tabular}{|c|c|c|c|c|c|}
\hline Age $<35$ Ongoing pregnancies, $\mathrm{n}(\%$ of transfer) & $21(25.0)$ & $73(33.2)$ & $53(48.6)$ & $147(35.6)$ & 0.001 \\
\hline Age (Years) $>35$ mean \pm SD & $37.4 \pm 1.5$ & $37.2 \pm 1.4$ & $36.9 \pm 1.5$ & $37.3 \pm 1.4$ & 0.105 \\
\hline Age $>35$ Ongoing pregnancies, $n$ (\% of started cycles) & $23(12.5)$ & $27(20.0)$ & $22(38.6)$ & $72(19.1)$ & 0.006 \\
\hline Age $>35$ Ongoing pregnancies, $n(\%$ of transfer) & $23(17.7)$ & $27(22.9)$ & $22(45.8)$ & $72(24.3)$ & 0.004 \\
\hline Cycles, $n \geq 2$ & $473(37.5)$ & $540(42.9)$ & $247(19.6)$ & $1260(100)$ & \\
\hline Age (Years) mean \pm SD & $35.4 \pm 3.6$ & $33.6 \pm 4.2$ & $32.5 \pm 4.1$ & $34.1 \pm 4.1$ & 0.001 \\
\hline Ongoing pregnancies, $\mathrm{n}$ (\% of started cycles) & $66(14.0)$ & $108(20.0)$ & $65(26.3)$ & $239(19.0)$ & 0.002 \\
\hline Ongoing pregnancies, $\mathrm{n}$ (\% of transfer) & $66(19.2)$ & $108(23.6)$ & $65(30.8)$ & $239(23.6)$ & 0.007 \\
\hline Age (Years) $<35$ mean \pm SD & $31.7 \pm 2.7$ & $31.0 \pm 3.1$ & $30.3 \pm 2.7$ & $31.0 \pm 3.0$ & 0.149 \\
\hline Age $<35$ Ongoing pregnancies, $n$ ( $\%$ of started cycles) & $35(17.8)$ & $71(21.3)$ & $53(30.6)$ & $159(22.6)$ & 0.009 \\
\hline Age $<35$ Ongoing pregnancies, $n$ ( $\%$ of transfer) & $35(23.0)$ & $71(25.1)$ & $53(37.6)$ & $159(27.6)$ & 0.008 \\
\hline Age (Years) $>35$ mean \pm SD & $38.0 \pm 1.2$ & $37.8 \pm 1.2$ & $37.6 \pm 1.2$ & $37.9 \pm 1.2$ & 0.213 \\
\hline Age $>35$ Ongoing pregnancies, $\mathrm{n}$ ( $\%$ of started cycles) & $31(11.2)$ & $37(18.0)$ & $12(16.2)$ & $80(14.4)$ & 0.101 \\
\hline Age $>35$ Ongoing pregnancies, $n$ ( $\%$ of transfer $)$ & $31(16.1)$ & $37(21.1)$ & $12(17.1)$ & $80(18.3)$ & 0.448 \\
\hline
\end{tabular}

1. P-value $<0.05$ was considered statistically significant.

2. Statistics, comment in text.

Table 4 shows the pregnancy rate the relationship to the first cycle and the second or more cycle based on AMH and age. In the first cycle, age did not affect pregnancy relative to $\mathrm{AMH}$ groups. The pregnancy rate was significantly high in groups with high $\mathrm{AMH}$, while in patients $>35$-years-old pregnancy rate was generally low, but still significantly high in groups with high AMH. In the second or more cycle of patients $\leq 35$-yearsold, age had no influence, and patients with high AMH had a high pregnancy rate. Patients $>35$-years-old in second or more cycle had no significant difference in pregnancy rate between $\mathrm{AMH}$ groups.

\section{Discussion}

The present study provides results from 2148 ART cycles where a stimulation based on the AMH level FSH dosing algorithm. The main findings of the present study were patients with AMH levels above $32 \mathrm{pmol} / \mathrm{L}$ and thus a predicted high rate of pregnancy. Several studies based on the number of oocytes that predict high pregnancy rates [16-19]. We know that patients with high AMH can develop more follicles, and as a result, many oocytes come with aspiration. It has always been difficult to keep the balance between having a high pregnancy and minimizing OHSS. Patients with anovulation have high AMH and tend to have many follicles on stimulation, but they may also have a low response if stimulated with an inadequate dose of FSH [20-22]. In our study we could show that patients with high $\mathrm{AMH}$, even some of them, do not have many oocytes nevertheless, had better pregnancy rate in the first cycle regardless of age. In patients >35-year-old, the rate of pregnancy decreases but is still present, for patients with high AMH has a high pregnancy rate compared to patients with low AMH.

Patients who had completed number two or more cycles pregnancy is still high in the group of patients with high AMH aged $<35$ years. In patients aged $>35$ years, with two or more cycles we could not find different inter-group pregnancy rates,

regardless of $\mathrm{AMH}$ level. AMH is a very good predictor of pregnancy prognosis. Patients with high AMH are at risk group for OHSS therefore we choose AMH algorithm to minimize OHSS and maintain good pregnancy rate. We have mildly stimulated patients with $\mathrm{AMH}>32$ where some of the patients have responded with very few follicles but the pregnancy rate was satisfactory and none of them had OHSS [23-25]. Several studies show that a number of oocytes 8-14 will provide better pregnancy rate [16-18]. We agree that patients with high $\mathrm{AMH}$ and young age can reach in most cases the number of oocytes we want. Our study confirms that AMH is a really good prediction for oocytes retrieval and pregnancy rate, especially in patients $<35$ years-old, whatever there is a first, second or more cycle.

Low AMH patients do not have a good pregnancy prognosis, the number of oocytes retrieval is not high and the pregnancy rate is low [26-30]. Our study shows that even though age was $<35$ years, the patients with low AMH had significant low pregnancy compared to patients with high AMH. AMH level reflects pregnancy chances in all groups, we can see differences in pregnancy rate in all AMH groups. Group of patients with $\mathrm{AMH}<12 \mathrm{pmol} / \mathrm{L}$ had poor pregnancy and group of patients with $\mathrm{AMH}>32 \mathrm{pmol} / \mathrm{L}$ had the best pregnancy rate. Patients with $\mathrm{AMH}$ $12-32 \mathrm{pmol} / \mathrm{L}$ normal responder stimulated with standard dose FSH, the pregnancy rate was better than the group of patients with low AMH, but low compared to the group of patients with high $\mathrm{AMH}>32 \mathrm{pmol} / \mathrm{L}$.

\section{Conclusion}

The main finding suggest that an $\mathrm{AMH}$ is a good predictor for pregnancy prognosis in patients at the least $<35$ years old. Patients with high AMH have a significantly better pregnancy rate compared to patients with low AMH. The AMH algorithm can be used to select the starting dose of FSH to minimize OHSS and retains good pregnancy rate. However, a randomised study with enough power with our protocol should be performed 


\section{Acknowledgement}

The authors wish to thank the staff at the Fertility Clinic, Rigshospitalet, for their role in data acquisition.

\section{References}

1. Dewailly D, Andersen CY, Balen A, Broekmans F, Dilaver N, et al. (2014) The physiology and clinical utility of anti-Mullerian hormone in women. Hum Reprod Update 20 (3): 370-385.

2. Seifer DB, Baker VL, Leader B (2011) Age-specific serum anti-Müllerian hormone values for 17,120 women presenting to fertility centers within the United States. Fertil Steril 95(2): 747-750.

3. Broekmans FJ, Kwee J, Hendriks DJ, Mol BW, Lambalk CB, et al. (2006) A systematic review of tests predicting ovarian reserve and IVF outcome. Hum Reprod Update 12(6): 685-718.

4. Nardo LG, Gelbaya TA, Wilkinson H, Roberts SA, Yates A, et al. (2009) Circulating basal anti-Müllerian hormone levels as predictor of ovarian response in women undergoing ovarian stimulation for in-vitro fertilization. Fertil Steril 92(5): 1586-1593.

5. Dorgan JF, Spittle CS, Egleston BL, Shaw CM, Kahle LL, et al. (2010) Assay reproducibility and within-person variation of Müllerian inhibiting substance. Fertil Steril 94(1): 301-304

6. Fleming R, Deshpande N, Traynor I, Yates RW (2006) Dynamics of FSH-induced follicular growth in subfertile women: relationship with age, insulin resistance, oocyte yield and anti-Mullerian hormone. Hum Reprod 21(6): 1436-1441.

7. Visser JA, Jong DFH, Laven JS, Themmen AP (2006) Anti-Müllerian hormone: a new marker for ovarian function. Reproduction 131(1): 1-9.

8. Marca LA, Sunkara SK (2014) Individualization of controlled ovarian stimulation in IVF using ovarian reserve markers: from theory to practice. Hum Reprod Update 20(1): 124-140.

9. Gomez R, Schorsch M, Hahn T, Henke A, Hoffmann I, et al. (2016) The influence of AMH on IVF success. Arch Gynecol Obstet 293(3): 667-673.

10. Broer SL, Broekmans FJ, Laven JS, Fauser BC (2014) Anti-Müllerian hormone: ovarian reserve testing and its potential clinical implications. Hum Reprod Update 20(5): 688-701.

11. Asada Y, Morimoto Y, Nakaoka Y, Yamasaki T, Suehiro Y, et al. (2017) Age-specific serum anti-Müllerian hormone concentration in Japanese women and its usefulness as a predictor of the ovarian response. Reprod Med Biol 16(4): 364-373.

12. Brodin T, Hadziosmanovic N, Berglund L, Olovsson M, Holte J, et al. (2013) Anti müllerian hormone levels are strongly associated with live-birth rates after assisted reproduction. J Clin Endocrinol Metab 98(3): 1107-1114.

13. Arce JC, Andersen AN, Fernández-Sánchez M, Visnova H, Bosch E, et al. (2014) Ovarian response to recombinant human follicle-stimulating hormone: a randomized, antimüllerian hormone-stratified, dose-response trial in women undergoing in-vitro fertilization/intracytoplasmic sperm injection. Fertil Steril 102(6): 1633-1640.

14. Nelson SM, Yates RW, Lyall H, Jamieson M, Traynor I, et al. (2009) Anti-Müllerian hormone-based approach to controlled ovarian stimulation for assisted conception. Hum Reprod 24(4): 867-875.

15. Yates AP, Rustamov O, Roberts SA, Lim HY, Pemberton PW, et al. (2011) Anti-Mullerian hormone-tailored stimulation protocols improve outcomes whilst reducing adverse effects and costs of IVF. Hum Reprod 26(9): 2353-2362.

16. Allegra A, Marino A, Volpes A, Coffaro F, Scaglione P, et al. (2017) A randomized controlled trial investigating the use of a predictive nomogram for the selection of the FSH starting dose in IVF/ICSI cycles. Reprod Biomed Online 34(4): 429-438.
17. Andersen NA, Nelson SM, Fauser BC, García-Velasco JA, Klein BM, et al. (2017) Individualized versus conventional ovarian stimulation for in-vitro fertilization: a multicenter, randomized, controlled, assessor-blinded, phase 3 noninferiority trial. Fertil Steril 107(2): 387-396.

18. Sunkara SK, Rittenberg V, Raine-Fenning N, Bhattacharya S, Zamora J, et al. (2011) Association between the number of eggs and live birth in IVF treatment: an analysis of 400135 treatment cycles. Hum Reprod 26(7): 1768-1774.

19. Magnusson Å, Källen K, Thurin-Kjellberg A, Bergh C (2018) The number of oocytes retrieved during IVF: a balance between efficacy and safety. Hum Reprod 33(1): 58-64.

20. Sopa N, Larsen EC, Andersen NA (2018) Low dose HP-hMG in an antagonist protocol for IVF in ovulatory and anovulatory patients with high AMH. Gynecol Endocrinol 34(7): 623-626.

21. Fauser BC, Diedrich K, Devroey P, Evian Annual Reproduction Workshop Group (2008) Predictors of ovarian response: progress towards individualized treatment in ovulation induction and ovarian stimulation. Hum Reprod Update 14 (1): 1-14.

22. DAmato G, Caringella AM, Stanziano A, Cantatore C, Palini S, et al (2018) Mild ovarian stimulation with letrozole plus fixed dose human menopausal gonadotropin prior to IVF/ICSI for infertile non-obese women with polycystic ovarian syndrome being pre-treated with metformin: a pilot study. Reprod Biol Endocrinol 16(1): 89.

23. Kissell KA, Danaher MR, Schisterman EF, Wactawski-Wende J, Ahrens KA, et al. (2014) Biological variability in serum anti-Müllerian hormone throughout the menstrual cycle in ovulatoryand sporadic anovulatory cycles in eumenorrheic women. Hum Reprod 29(8): 1764-1772.

24. Olivennes F, Trew G, Borini A, Broekmans F, Arriagada P, et al. (2015) Randomized, controlled, open-label, non-inferiority study of the CONSORT algorithm for individualized dosing of follitropin alfa. Reprod Biomed Online 30(3): 248-257.

25. Sterrenburg MD, VeltmanVerhulst SM, Eijkemans MJ, Hughes EG, Macklon NS, et al. (2011) Clinical outcomes in relation to the daily dose of recombinant follicle-stimulating hormone for ovarian stimulation in in-vitro fertilization in presumed normal responders younger than 39 years: a meta-analysis. Hum Reprod Update 17(2): 184-196.

26. Yakin K, Oktem O, Balaban B, Urman B (2018) Bologna criteria are predictive for ovarian response and live birth in subsequent ovarian stimulation cycles. Arch Gynecol Obstet.

27. Aboulghar M, Saber W, Amin Y, Aboulghar MM, Serour G, et al. (2014) Impact of antimüllerian hormone assays on the outcomes of in vitro fertilization: a prospective controlled study. Fertil Steril 101(1): 134137.

28. Grzegorczyk-Martin V, Khrouf M, Bringer-Deutsch S, Mayenga JM, Kulski O, et al. (2012) Low circulating anti-Müllerian hormone and normal follicle stimulating hormone levels: which prognosis in an IVF program? Gynecol Obstet Fertil 40(7-8): 411-418.

29. Taronger R, Martínez-Cuenca S, Ferreros I, Rubio JM, Fernández-Colom PJ, et al. (2018) Ovarian stimulation with corifollitropin alfa followed by hp-hMG compared to hp-hMG in patients at risk of poor ovarian response undergoing ICSI: A randomized controlled trial. Eur J Obstet Gynecol Reprod Biol 231: 192-197.

30. Sefrioui O, Madkour A, Aboulmaouahib S, Louanjli KIN (2018) Women with extreme low AMH values could have in vitro fertilization success. Gynecol Endocrinol 16:1-4. 
(C) (P) This work is licensed under Creative (C) Commons Attribution 4.0 License BY DOI: 10.19080/JGWH.2019.13.555872
Your next submission with Juniper Publishers will reach you the below assets

- Quality Editorial service

- Swift Peer Review

- Reprints availability

- E-prints Service

- Manuscript Podcast for convenient understanding

- Global attainment for your research

- Manuscript accessibility in different formats ( Pdf, E-pub, Full Text, Audio)

- Unceasing customer service

Track the below URL for one-step submission https://juniperpublishers.com/online-submission.php 